\title{
Applications of Near Infrared Spectroscopy in Neurorehabilitation
}

\author{
Masahito Mihara and Ichiro Miyai \\ Neurorehabilitation Research Institute, Morinomiya Hospital \\ Japan
}

\section{Introduction}

In developed countries, stroke is a major cause of acquired disability among adults. Although there is a considerable inter-subject variability, the time course of functional recovery assumes an exponential shape, with a faster recovery in the initial few weeks, followed by a slower recovery over the next few months (Jorgensen et al., 1999; Duncan et al., 2000). In the former phase, faster recovery is thought to be due to the reduction of parenchymal oedema or recanalization of the blood flow. The latter phase is believed to depend upon the adaptive plasticity of the brain, including unmasking or disinhibiting the potentially aberrant neural network, and vicariation of function (Ward \& Frackowiak, 2004). Although there are many evidences for brain plasticity after stroke or brain injury, most of our knowledge is derived from animal experiments (Jenkins \& Merzenich, 1987; Nudo et al., 1996). Direct investigation of functional reorganization after brain damage in humans has only recently become possible with advancements in non-invasive functional imaging techniques, such as positron emission tomography (PET) and functional MRI (fMRI). Among these functional neuroimaging techniques, functional near infrared spectroscopy (NIRS) has drawn attention from investigators in rehabilitation medicine since it is thought to be less constrained and more available for measurement during various tasks. In this chapter, we introduce the clinical applications of fNIRS in the field of rehabilitation medicine and I shall discuss the further possibilities for its application.

\section{Application of functional NIRS in studies of human motor control}

\subsection{Principles of functional NIRS}

Near infrared light, particularly that with a wavelength between 700 and $900 \mathrm{~nm}$, can easily pass through biological tissues, including skin and skull bone, and be absorbed by biological chromophores such as haemoglobin, myoglobin, and cytochrome oxidase in the mitochondria. Because myoglobin concentration is much lower than haemoglobin concentrations in the brain tissue and a change in the redox state of cytochrome oxidase occurs only under severely hypoxic conditions, near infrared light is mainly absorbed by haemoglobin when used as a functional brain-imaging tool. The NIRS system with continuous waves, which is widely used in commercially available instruments, measures the transmitted intensity and calculates the relative changes in the haemoglobin concentration according to the modified Beer-Lambert law for highly scattering media 
(MBLL) (Cope et al., 1988). If the light attenuation by scattering is considered constant, MBLL is denoted as follows:

$$
\Delta A_{\lambda 1}=\left(\varepsilon_{\lambda 1}^{\text {OxyHb }} \cdot \Delta C^{O x y H b}+\varepsilon_{\lambda 1}^{\text {DeoxyHb }} \cdot \Delta C^{\text {DeoxyHb }}\right) \cdot L
$$

Where $\varepsilon_{\lambda}$ is the extinction coefficient at a given wavelength $\lambda$, L is the optical pathlength, and $\Delta \mathrm{C}$ is the change in the concentration of each chromophore. If measurements with multiple wavelengths are performed simultaneously and optical pathlength is considered to be constant across the measurement, the product of the change in concentration of the chromophore and the optical pathlength can be calculated by solving the simultaneous equations. However, it should be noted that the precise optical pathlength is difficult to determine with the continuous-wave NIRS system. Therefore, calculated measurements are usually denoted in arbitrary units such as millimolar millimetres $(\mathrm{mM} \times \mathrm{mm})$ (Maki et al., 1995). It is generally accepted that the distribution of near infrared light paths between an illuminator-detector pair become 'banana-shaped' (Gratton et al., 1994), and that certain interoptode distances are needed for the propagation of near infrared light to the cerebral cortex. Commonly, a distance of $2 \mathrm{~cm}$ or more is used.

In the brain tissues, regional brain activation is accompanied by an increase in the regional blood flow (Fox \& Raichle, 1986), and this regional blood flow increase is thought to exceed the regional oxygen consumption. Therefore, regional cortical activation results in a regional increase in the oxygenated haemoglobin $(\mathrm{OxyHb})$ levels, with a decrease in the deoxygenated haemoglobin $(\mathrm{DeoxyHb})$ levels. Similar to fMRI or PET, functional NIRS detects the task-related haemodynamic responses, that is, the task-related increase in the $\mathrm{OxyHb}$ signal and/or the task-related decrease in DeoxyHb.

\subsection{Potential advantages and shortcomings of functional NIRS}

There are several potential advantages of functional NIRS for investigating human brain activity. First, functional NIRS imposes less onerous constraints on its subjects. In a NIRS system, minor head and truncal motion is irrelevant, if a tight contact is maintained between the skull surface and optodes during measurement. Second, unlike other neuroimaging modalities, functional NIRS requires relatively small and simple equipment. Finally, the NIRS system is completely safe and non-invasive, since it uses only a low-power near infrared laser. It therefore enables us to investigate brain activation under natural conditions, such as at the bedside or while sitting on a chair, and measure cortical activation in the activities of daily life, such as standing and walking. Based on these characteristics, NIRS is thought to be a suitable neuroimaging tool for clinical investigation in fields such as paediatric neurology and rehabilitation medicine.

Despite these potential advantages, NIRS has several shortcomings as a functional neuroimaging tool. First, NIRS cannot measure activation in deep brain structures, including the basal ganglia, brainstem, and cerebellum. Secondly, NIRS has relatively poor spatial resolution (a few centimetres) and cannot provide any spatial information. Therefore, spatial registration should be made with other data, such as anatomical information from MRI scans and real-world coordinates derived from a 3-dimensional digitizer and other standard references (Okamoto et al., 2004). Third, with the continuous-wave NIRS system, we cannot measure the precise optical pathlength, and therefore cannot measure absolute 
changes, but only relative changes, in the haemoglobin concentration. Finally, not only the brain tissue but also skin tissue beneath the optodes can affect NIRS signal changes. To avoid or cancel the effect of skin blood flow, several methodologies have been introduced (Kohno et al., 2007; Yamada et al., 2009), but there is no 'gold standard' for this problem. Therefore, researchers should be cautious about the contamination of these non-brain signals when interpreting NIRS measurements (Takahashi et al., 2011).

\subsection{Application of NIRS to human gait control}

Gait requires complex visuo-sensorimotor coordination. Like in other animals, human locomotion is controlled by multiple neural systems, hierarchically distributed throughout the central nervous system, including the spinal cord, brainstem, cerebellum, basal ganglia, and motor cortex (Grillner \& Wallen, 2004). Although most studies of neuronal mechanisms of gait control were conducted with quadruped animals, a bipedal stance and gait are unique functions of humans. Therefore, functional imaging studies in humans are important for investigating the neural mechanisms of gait control. However, as stated above, it is difficult to study dynamic movements such as gait control with conventional neuroimaging techniques, and functional NIRS is a suitable tool for these studies.

\subsubsection{Cortical activation of gait in healthy subjects}

Using a multi-channel NIRS system, Miyai and colleagues reported cortical activation during human gait on a treadmill (Miyai et al., 2001). They used a custom-made plastic holder cap and a weight-balancing system to avoid excessive motion artifacts during the locomotor task, and they could measure the task-related haemoglobin signal changes from the frontoparietal skull surface (Fig. 1).

A

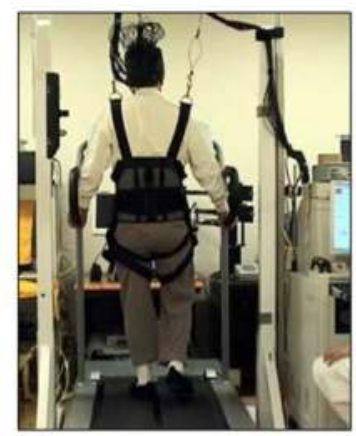

B

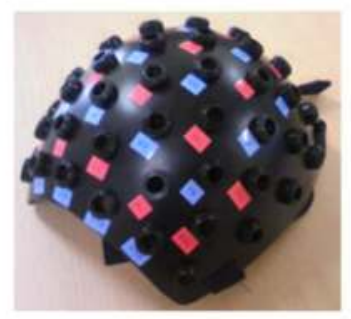

C

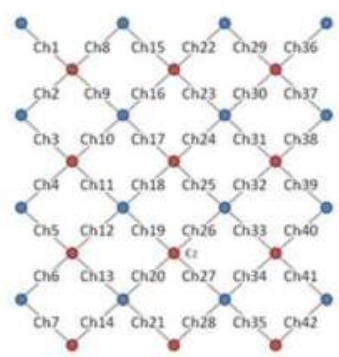

A: Subject performing a locomotor task on the treadmill. B: A custom-made plastic holder cap for fixing optode fibres. C: The schematic location of each optode and channel. Cz represents the vertex. Red and blue circles represent the light sources and detector fibres.

Fig. 1. Measurement of cortical activation during walking by using a functional NIRS system

In healthy subjects, the locomotor task on a treadmill evoked symmetrical activation in the medial sensorimotor cortex and supplementary motor area (Fig. 2). These findings were consistent with results from a study using single photon emission tomography (Fukuyama 

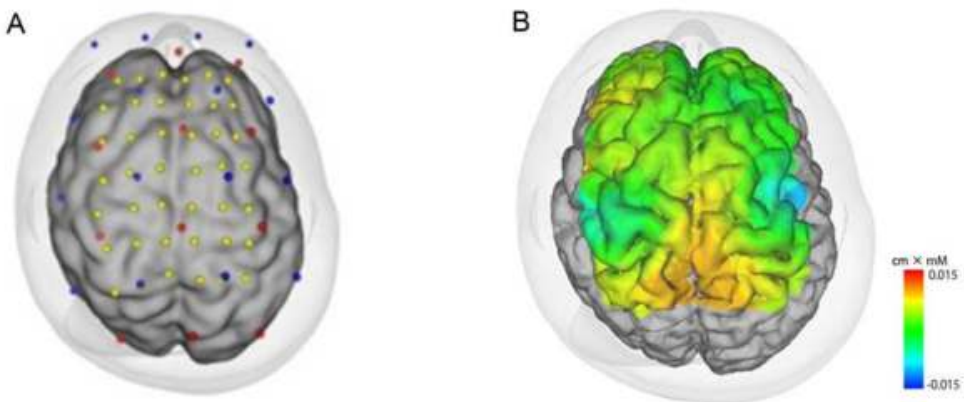

A: Optode locations on the skull surface (red and blue dots) and estimated cortical projection points of functional NIRS measurement (yellow dots) B: Cortical activation map based on OxyHb signal changes during $3-\mathrm{km} / \mathrm{h}$ gait on the treadmill. Data averaged from 3 repetitions of 30-s gait followed by 30 -s rest periods.

Fig. 2. Cortical activation during gait in a healthy subject

et al., 1997). Furthermore, it was suggested that the different areas of the cerebral cortex play different roles in human gait control. During the locomotor task, cortical activation in the prefrontal cortex temporally increased during the acceleration phase of gait, and gradually decreased during walking at a stable speed (Suzuki et al., 2004). It was also revealed that cortical activation change was more prominent at higher locomotor speed in the prefrontal cortex, but cortical activation in the sensorimotor cortex was not associated with gait speed. Therefore, the prefrontal cortex was involved in the adaptation of gait speed, but the medial sensorimotor cortex was involved in stable gait.

\subsubsection{Cortical activation of gait in stroke patients}

Gait disturbance is a major consequence of stroke and is shown to greatly affect the ability to independently perform activities of daily living (ADL). Although it is assumed that functional reorganization of the central nervous system plays an important role in gait recovery after stroke, there is not enough evidence to associate cortical reorganization with gait recovery. Because the NIRS system is non-invasive and places fewer burdens on the patient, it is useful for repetitive measurement of the cortical activation of gait. In stroke patients, there are several reported cortical activation patterns. In hemispheric stroke patients with supratentorial lesions, activation in the motor-related cortex in the affected hemisphere increases with functional recovery. Particularly, the premotor cortex in the affected hemisphere appears to be essential (Miyai et al., 2002, 2003), as a previous observational study had suggested (Miyai et al., 1999). On the other hand, ataxic stroke patients with infratentorial lesions display a different activation pattern during gait. In ataxic stroke patients, activation in the medial sensorimotor cortex is not significantly changed compared to that in the healthy subjects. However, the prefrontal activation pattern differs, and ataxic patients show sustained activation in the prefrontal cortex (Fig. 3) (Mihara et al., 2007). As described above, prominent activation in the prefrontal cortex is reported in the acceleration phase of gait in healthy subjects, and is presumed to be involved in the adaptation of gait speed. Ataxic stroke patients exhibit increased postural sway, difficulty in multi-joint adjustment during locomotion, and reduced walking speed (Morton \& Bastian, 


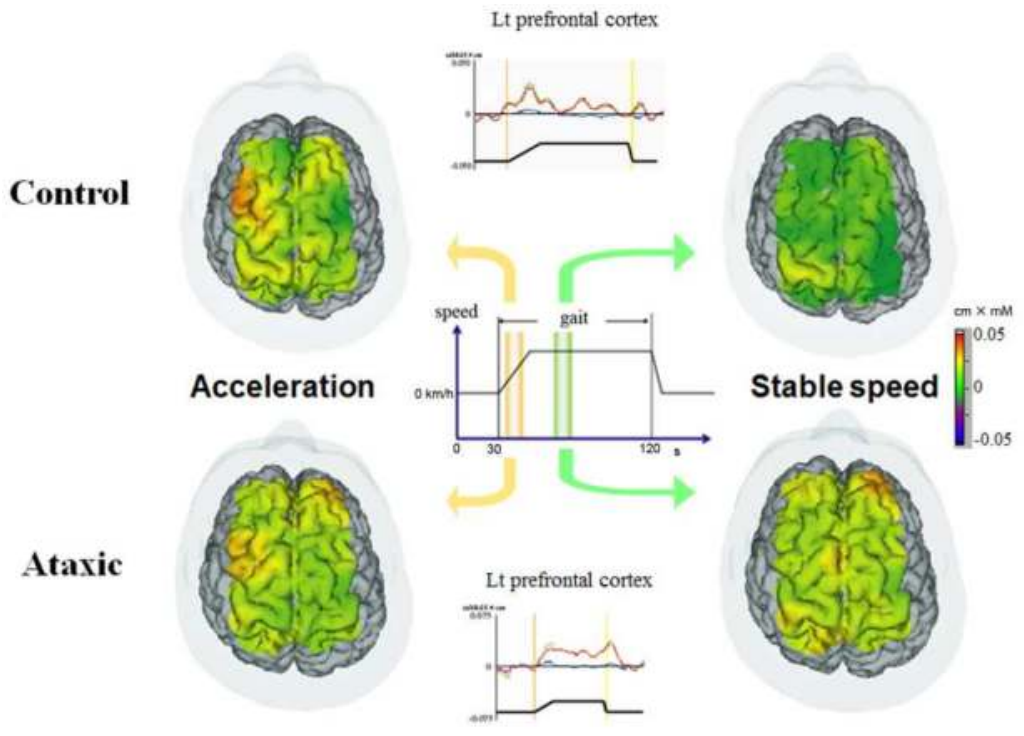

Cortical mapping results from healthy control subjects and ataxic stroke patients. In the acceleration phase, the cortical activation in both groups was consistent, but in the stable phase, cortical activation (particularly activation in the prefrontal cortex) was sustained in ataxic stroke patients but reduced in healthy control subjects. Raw data from the NIRS channel covering the left prefrontal cortex is also shown (red, blue, and green lines denote Oxy-, Deoxy-, and total-Hb signal changes, respectively).

Fig. 3. Gait-related cortical activation in the healthy and ataxic stroke patients

2004). Such a substantial variability of limb movements may require both more attention and more intention to control lower limb movements during gait. Thus, our findings are in accordance with the hypothesis that the impaired control of automated locomotion in ataxic patients is compensated by recruitment of the frontal cortices in the course of gait recovery, especially the prefrontal cortex.

\subsubsection{Supposed mechanisms for the gait recovery after stroke}

The abovementioned NIRS studies revealed the vital role of cortical reorganization in gait recovery after stroke, and that the cortical activation pattern could differ with the lesion location and size. One possible interpretation for these findings is that a widespread neural network is engaged in the locomotor control, and different regions regulate different aspects. Although both the infra- and supratentorial structures are thought to regulate locomotion through the putative central pattern generator in the spinal cord (Dimitrijevic et al., 1998), these contribute to different aspects of locomotor control. The infratentorial regions, including the reticular nuclei and the medial cerebellum, are regarded as the main structures for controlling automated locomotion and muscle tone during gait (Armstrong, 1988; Drew et al., 2004; Mori et al., 2004). In contrast, the supratentorial structures are predominantly involved in adjusting locomotor performance to an altered environment (Armstrong, 1988; Matsuyama et al., 2004; Takakusaki et al., 2004). For instance, studies using fMRI and PET report decreased the cortical activation during motor imaging of 
automated locomotion, such as walking at a steady speed (Malouin et al., 2003; Jahn et al., 2004). These results indicate that the cortical demand for controlling gait is reduced during the steady phase of locomotion in control subjects, since the infratentorial structures engage in automated locomotion.

In patients with hemiparetic stroke and supratentorial lesion, it is assumed that the infratentorial locomotor centre, which engages in automated locomotion, is intact. Therefore, increased activation in the ipsilesional premotor cortex, which has ample connections to the brainstem and basal ganglia, is likely to compensate for the reduced regulatory output from the cerebral cortex. In contrast, automated locomotor control is impaired in ataxic stroke patients with infratentorial lesions. Thus, continuous monitoring and adjustment are needed, and this may result in sustained activation of the prefrontal cortex (Fig. 4).

A

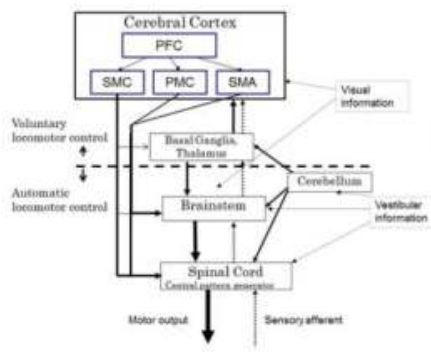

B

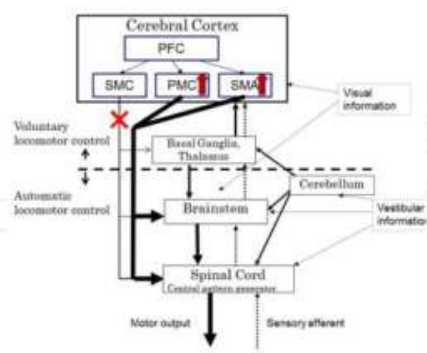

C

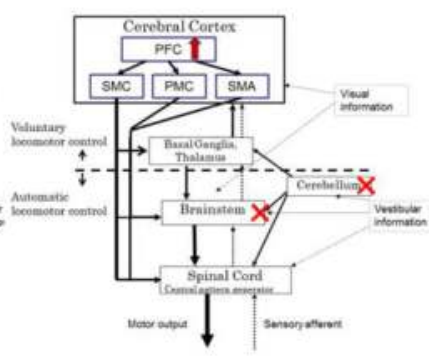

A: Neural network in central nervous system for locomotor control B: Assumed compensatory reorganization for gait recovery in supratentorial hemiparetic stroke C: Assumed compensatory reorganization for gait recovery after infratentorial ataxic stroke

Fig. 4. Suggested framework for locomotor recovery after stroke

\subsection{Cortical activation associated with the maintenance of postural balance}

Our habitual bipedal stance is highly advantageous because it enables us to maximize the dexterity of our hands, which is useful for developing tools. However, as a trade-off for this advantage, the unstable nature of bipedal standing increases the tendency to fall, leading to severe injuries such as limb fracture, joint dislocation, and head injury. A community-based study revealed that more than one-third of the people aged over 65 years fell at least once a year, and that proportion increased to $50 \%$ by the age of 80 years (O'Loughlin et al., 1993). To prevent falls and resultant disability in the elderly, it is essential to understand the neural mechanisms underlying bipedal standing. Accumulated results from studies in cats and other vertebrates (Armstrong, 1988; Drew et al., 2004) suggest that multiple automated and reflexive actions regulated by the subcortical structures contribute to balance control. However, since balance control in bipedal standing requires more complex and sophisticated sensorimotor coordination than quadruped standing, it is plausible that not only the subcortical structures but also the well-evolved cerebral cortices are involved in balance control during bipedal standing in humans (Nielsen, 2003). In line with this notion, recent studies suggest that the cerebral cortex is involved in human balance control (Dietz et al., 1984; Quant et al., 2005; Slobounov et al., 2005). Compared to conventional neuroimaging techniques, such as fMRI or 
PET, functional NIRS is relatively robust against a subject's motion and seems to be suitable for investigating cortical involvement in postural control.

\subsubsection{Cortical activation with postural perturbation in healthy subjects}

Using functional NIRS, Mihara et al. reported the cortical activation associated with predictable and unpredictable postural perturbation (Mihara et al., 2008). During the experiment, subjects were asked to stand still on a platform, and postural perturbation was given by the brisk forward and backward translation of the platform (Fig.5). Oxy- and DeoxyHb signal changes were recorded from 50 channels on the frontoparietal skull surface with a sampling rate of $4 \mathrm{~Hz}$.
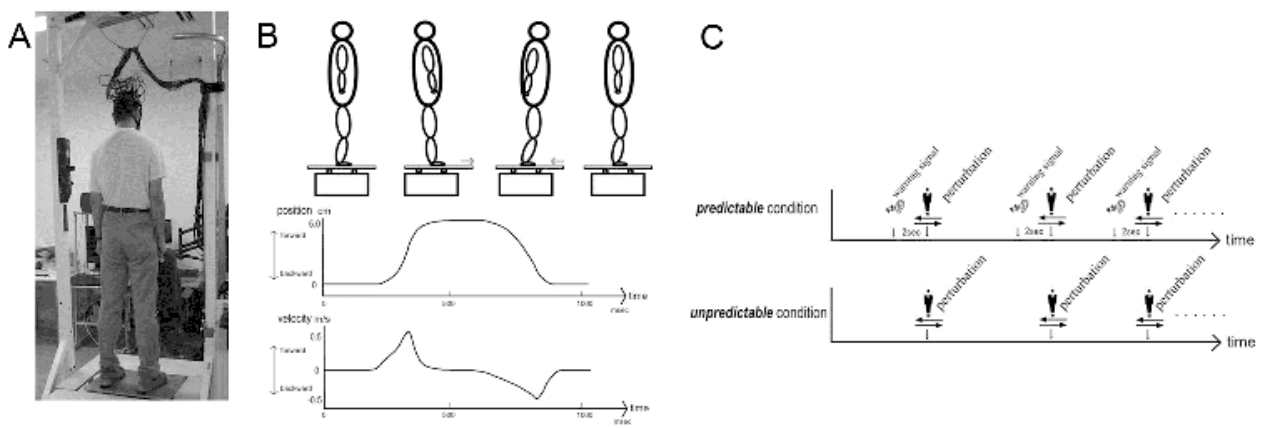

A: Experimental overview: Subjects are asked to stand on the custom-made platform with their feet shoulder-width apart. B: Schematic figure of platform movement. The middle and bottom row denote the time course of platform position and velocity. C: Task protocol in both conditions. Postural perturbations are given with randomised intervals. In the predictable condition, auditory warning signals are provided $2 \mathrm{~s}$ before the perturbation.

Fig. 5. Experimental setup for the measurement of cortical activation associated with postural perturbation.

Statistical analyses, using a general linear model with least squares estimation, revealed significant task-related $\mathrm{OxyHb}$ increase in the bilateral prefrontal cortex regardless of preceding warning cues. In the predictable condition, the supplementary motor area and the parietal association cortex were activated as well as the prefrontal cortex (Fig. 6).

Considering that results from previous studies imply attentional demands for postural control (Woollacott \& Shumway-Cook, 2002), the prefrontal involvement suggests the recruitment of the attentional process for the maintenance of standing posture against postural perturbation. The supplementary motor area and the parietal association cortex, however, were more activated with the preceding warning cues, suggesting that these cortices are involved in voluntary postural control.

\subsubsection{Possible application of functional NIRS as a surrogate marker for balance ability}

Neurological disorders, including stroke, Parkinson's disease, and spinocerebellar ataxia, affect the balance ability and activity of daily living. In the field of rehabilitation medicine, 

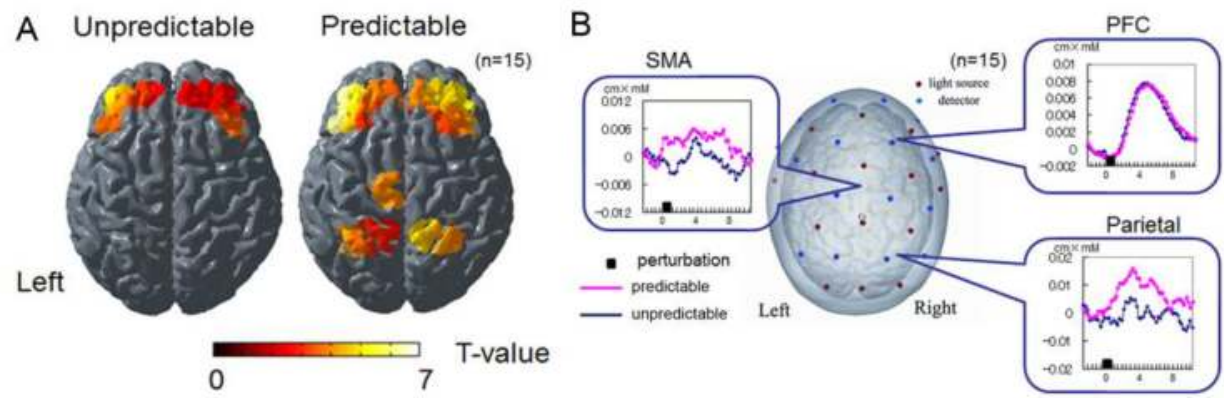

A: Cortical activation mapping based on task-related $\mathrm{OxyHb}$ signal increase. In the unpredictable condition, a task-related $\mathrm{OxyHb}$ signal increase is prominent in the bilateral prefrontal cortex (left). In the predictable condition, postural perturbation evoked task-related OxyHb signal increases in the supplementary motor area and parietal association cortex, as well as the bilateral prefrontal cortex (right). B: Timeline analyses of the OxyHb signal in both conditions.

SMA: supplementary motor area, PFC: prefrontal cortex

Fig. 6. Cortical activation associated with postural perturbation in healthy subjects.

balance impairment is one of the main targets for improvement. As described above, functional NIRS can monitor the activation of the cortical network, which is vital for postural control. Therefore, functional NIRS may possibly help objectively monitor balance recovery. Below, we present several cases in which we observe changes in cortical activation during functional recovery after inpatient rehabilitation.

A hemiplegic stroke patient with a subcortical lesion showed a similar cortical activation pattern change. Figure 7 shows the longitudinal change of cortical activation mapping associated with unpredicted postural perturbation. In a left hemiplegic patient, activation in the broad cortical area, including the bilateral prefrontal cortex, premotor cortex,

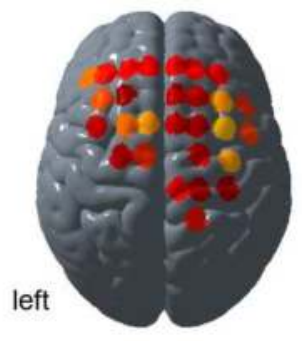

$122 \mathrm{~d}$ after onset

Berg Balance Scale: 26/56 pontine hemorrhage

45 y.o. male

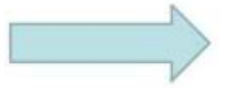

rehabilitation

t-value

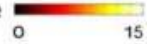

$166 \mathrm{~d}$ after onset

Berg Balance Scale: $46 / 56$

Cortical activation mapping based on task-related $\mathrm{OxyHb}$ signal increase associated with unpredictable postural perturbation. Cortical activation was increased after $40 \mathrm{~d}$ of inpatient rehabilitation.

Fig. 7. Cortical mapping change associated with postural perturbation in a patient with left hemiplegic stroke (45 years old). 
supplementary motor area, and sensorimotor cortex, became evident as his balance ability recovered after inpatient rehabilitation. These results suggest that balance recovery is associated with cortical activation change, as is upper and lower limb recovery after stroke (Calautti \& Baron, 2003; Ward et al., 2003; Luft et al., 2005; Enzinger et al., 2009).

Interestingly, not only stroke patients show functional recovery-associated cortical mapping pattern changes. Figure 8 shows cortical activation with postural perturbation in a 73-yearold male patient with Parkinson's disease. He exhibited postural instability and could not walk without assistance at admission, but after 4 weeks of inpatient rehabilitation and drug control, his condition improved and he became ambulant. Postural perturbation-related cortical activation was dramatically changed along with functional recovery. Cortical activation in the bilateral prefrontal, premotor, and supplementary motor areas was increased after inpatient rehabilitation. Considering the subcortical nature of Parkinson's disease, increased cortical activation may compensate for subcortical dysfunction, similar to gait in the ataxic stroke patients described above. Although there are several issues to be elucidated, these results imply that functional NIRS could be used as a surrogate marker for balance ability in patients with neurological disorders.

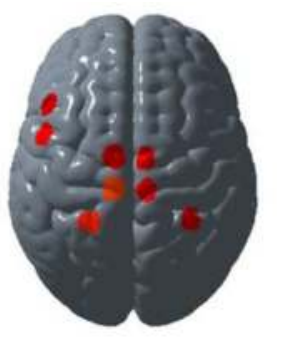

rehabilitation \& drug control
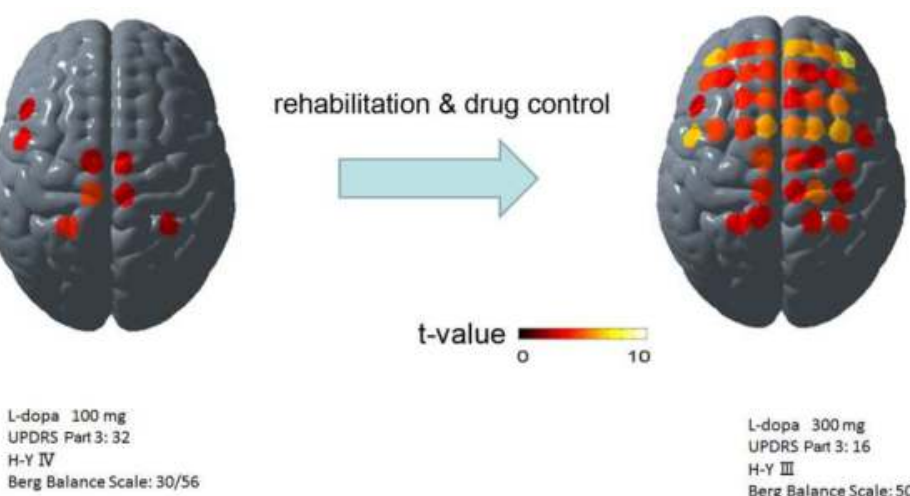

$$
\begin{aligned}
& \text { L-dopa } 300 \mathrm{mg} \\
& \text { UPDRS Part 3:16 } \\
& \text { H-Y III } \\
& \text { Berg Balance Scale: } 50 / 56
\end{aligned}
$$

Cortical activation mapping based on the task-related $\mathrm{OxyHb}$ signal increase associated with unpredictable postural perturbation. Cortical activation increased along with the recovery of balance ability in a widespread cortical area, including the bilateral prefrontal, premotor, and supplementary motor areas.

Fig. 8. Cortical mapping change associated with postural perturbation in a patient with Parkinson's disease (73 years old).

\subsection{Functional NIRS as a tool for motor learning studies}

Motor learning is vital and essential process in acquiring motor skills in daily life, not only for the neurological patients, but also for healthy individuals. Motor learning comprises motor sequence learning and motor adaptation in experimental settings (Doyon \& Benali, 2005). Motor sequence learning is assessed by the incremental acquisition of movements with repetition, while motor adaptation refers to the ability to compensate for environmental changes. To date, most human studies of motor sequence learning have used positron emission tomography (PET) or functional magnetic resonance imaging (fMRI) and 
investigated the neural mechanisms underlying the learning of sequential movements of fingers or feet in a lying position. However, motor learning usually occurs under postural control since most movements are executed while subjects are sitting or standing in daily situations. As described above, because of less postural restriction, functional NIRS is considered suitable for investigating motor learning under normal daily circumstances.

Using functional NIRS, Hatakenaka et al. studied the cortical activation change during the pursuit rotor (PR) task in healthy subjects (Hatakenaka et al., 2007). A pursuit rotor (PR) is a tool to evaluate motor sequence learning by measuring the ability to keep a stylus on a rotating target. In the PR task, there is no need for precise control of finger movements, but it requires motor control of proximal parts of the upper extremity, including the shoulder and elbow, as well as postural control for sitting (Grafton et al., 1992).In this study, 18 righthanded healthy subjects performed 8 repetitions of 30-s PR tasks followed by 30-s rest periods. Gains of motor skill were evaluated by the contact time between the stylus and target. During 8 repetitions of the PR task, cortical activation in the frontoparietal cortices was evaluated with functional NIRS.

As shown in Figure 9, performance of the subjects improved with PR task repetition. A taskrelated $\mathrm{OxyHb}$ increase was observed in the sensorimotor cortex, prefrontal cortex, and premotor cortex. A task-related DeoxyHb decrease was also observed. Interestingly, the centre of the task-related $\mathrm{OxyHb}$ increase was observed in the pre-supplementary motor area initially, but it shifted caudally to the supplementary motor area with cycle repetitions (Fig. 9). These data suggest that the pre-supplementary motor area and the supplementary motor area play different roles in the motor skill learning process.

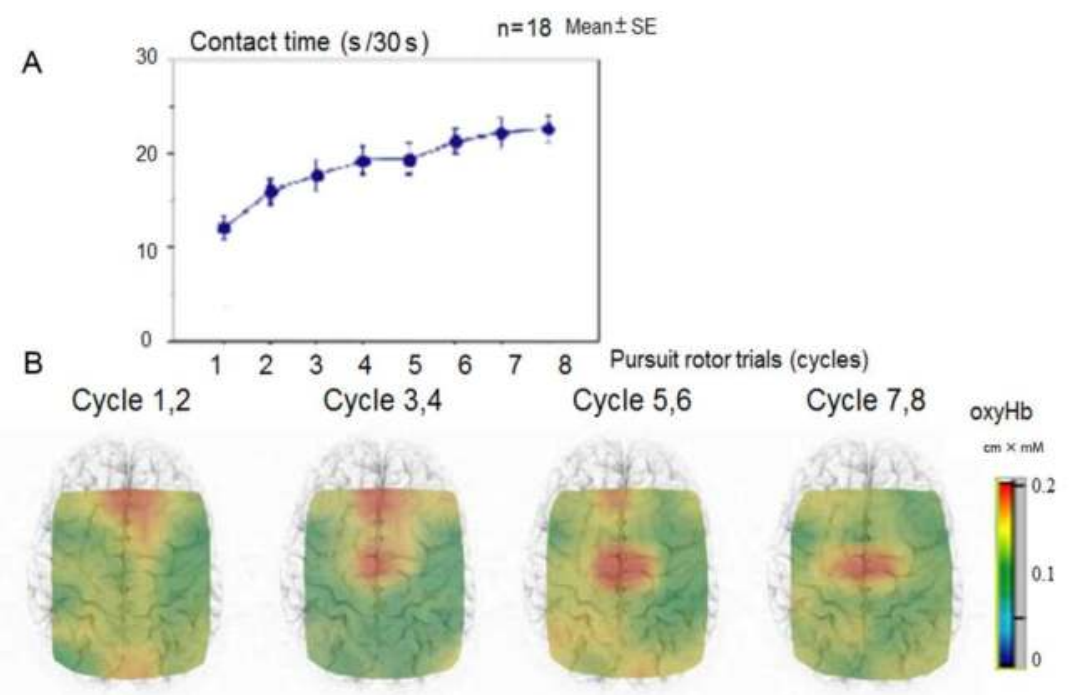

A: Cycle-by-cycle contact time during the pursuit rotor (PR) task in healthy right-handed subjects. B: Cortical activation mapping based on the task-related $\mathrm{OxyHb}$ signal increase associated with the PR task in a representative subject. A caudal shift of the centre of task-related cortical activation from the pre-supplementary motor area to the supplementary motor area was evident.

Fig. 9. Cortical mapping change associated with motor sequence learning. 
Functional NIRS was also used in a study investigating the adaptation learning process. We investigated the cortical activation changes during the adaptation of reaching with robotic devices with 3-dimensional virtual reality (VR) system (Mihara et al., 2009), in which the limb coordination pattern is altered. Seven right-handed healthy subjects participated in this study. An upper limb training system, mediated by electro-rheological fluid actuators with $3^{\circ}$ of free movement range, was used (Furusho et al., 2005). The subjects are given visual feedback of the position and the movement of the gripping handle of the robot arm, as the coloured ball (object) moves in the 3-dimensional VR space on the 13"monitor. The experiment consisted of 16 cycles of alternating 12-s rest and 8-s task periods. In the rest period, the floating target (Target) was fixed at the near lower left corner (the home position) of the VR space. In the reaching task period, the Target moved from the home position to the far upper right corner, and then returned to the home position in $8 \mathrm{~s}$, and they asked the subject to follow the Target by moving the robot arm. The distance between the Object and the Target was indicated by the colour of the object. The mean distance in each cycle was calculated as a measure of performance. Cortical activation was measured as $\mathrm{OxyHb}$ signal change using 50-channel functional NIRS from the frontoparietal area, and the task-related cortical activation of each subject was modeled using 2 orthogonal covariates (Buchel et al., 1998). The first was the task covariate, modeled as a boxcar function in all task periods, and the second was the error covariate that comprised a boxcar function scaled by the mean distance. The error covariate was mean-corrected and orthogonalized with respect to the first covariate. Both covariates were convolved with the canonical haemodynamic response functions and used in a GLM analysis. Group analysis was performed using the random effects model.

The mean distance of 7 subjects gradually reduced with cycle repetitions, indicating that the subjects learned to adapt to the visuo-motor reaching task in the VR space. Group analysis of functional NIRS signals showed significant effects primarily in the bilateral prefrontal, bilateral premotor, and left primary sensorimotor areas. Significant negative correlations to the error covariate were also found in the left prefrontal and premotor areas (Fig. 10). Although a broad cortical network is involved in the visuo-motor adaption learning of the reaching task, the prefrontal and premotor areas may be involved in the early stages of the adaptation process.

\subsection{Future directions of functional NIRS in the rehabilitation field}

To improve the performance of the activities of daily life and to restore function in severely damaged patients with stroke or neurodegenerative disease, there has been much interest in developing brain-computer interface (BCI) technology (Dobkin, 2007; Daly \& Wolpaw, 2008). The main purpose of BCI development is to substitute for lost neuromuscular output. For interactions between the brain and the outer environment, the BCI system must be able to detect brain signal, decode these signals, and output the appropriate information. Among several techniques for detecting brain activity, functional NIRS has attracted attention because of its portability and noninvasiveness. Although the clinical usage of BCI in the rehabilitation field is limited at this time, decoding techniques are improving with enthusiastic efforts from many groups. Sitaram et al. reported that right/left finger motor imagery could be classified with an average of $89 \%$ accuracy by applying a pattern recognition algorithm with hidden Markov Models to the multi-channel NIRS data 
A
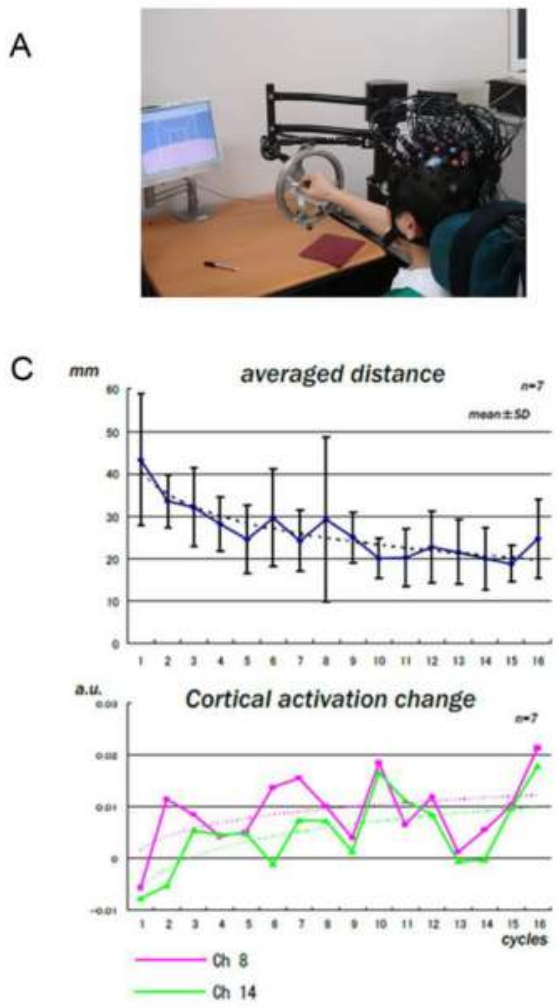

B

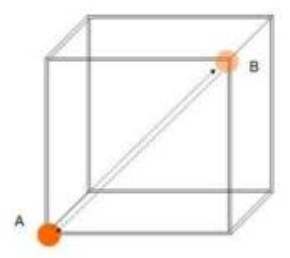

\section{Dorsal premotor cortex (ch 8 \& ch 14)}

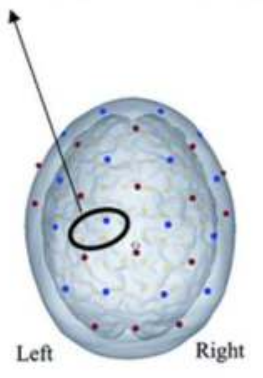

A: Experimental setting of the robotic rehabilitation system with functional NIRS. B: Reaching task with the robotic rehabilitation system. In a virtual reality space, subjects were asked to follow the target, which moves from the lower left corner to the upper right corner, and then back to the lower left corner in $8 \mathrm{~s}$. C: Average distance from the handle position and the target, and cortical activation in the contralateral premotor cortex.

Fig. 10. Adaptation learning task with the 3-dimensional robotic rehabilitation system

(Sitaram et al., 2007). As another approach, the real-time analysis of the NIRS signal with an adaptive general linear model using Kalman filtering was also reported (Abdelnour \& Huppert, 2009).

In addition, BCI could be used as a tool to augment cortical plasticity. Using electroencephalography signals, it has been shown that providing feedback of brain activity to the subjects can alter their brain activity itself. This technique, known as 'neurofeedback', has been investigated for several years and is already used in the clinical setting. It has been reported that real-time feedback of EEG activity enables voluntary regulation of cortical activation and attentional level (Delorme \& Makeig, 2003), and this has been applied in the treatment of attention deficit and hyperactivity disorder (Fuchs et al., 2003) and epilepsy (Kotchoubey et al., 2001). Combined with the real-time signal processing technique described above, functional NIRS could be used as a neurofeedback system for enhancing cortical plasticity. 


\section{Conclusion}

Functional NIRS is a unique tool as a neuroimaging modality. Despite several shortcomings, including difficulty in measuring haemoglobin oxygenation changes in deep brain structures and poor spatial resolution, it has characteristic advantages that can measure cortical activation in normal daily conditions. Functional NIRS could be used not only as a tool for measurement of cortical activation but also as a tool for treatment with further advancements in data analysis techniques.

\section{References}

Abdelnour, A. F. \& Huppert, T. (2009).Real-time imaging of human brain function by nearinfrared spectroscopy using an adaptive general linear model.Neuroimage, Vol.46,No. 1, pp 133-43

Armstrong, D. M. (1988).The supraspinal control of mammalian locomotion.J Physiol, Vol.405, p 1-37

Buchel, C., Holmes, A. P., Rees, G. \&Friston, K. J. (1998). Characterizing stimulus-response functions using nonlinear regressors in parametric fMRI experiments. Neuroimage, Vol.8,No. 2, pp 140-8

Calautti, C. \& Baron, J. C. (2003). Functional neuroimaging studies of motor recovery after stroke in adults: a review. Stroke, Vol.34,No. 6, pp 1553-66

Cope, M., Delpy, D. T., Reynolds, E. O., Wray, S., Wyatt, J. \& van der Zee, P. (1988). Methods of quantitating cerebral near infrared spectroscopy data.AdvExp Med Biol, Vol.222,, p 183-9

Daly, J. J. \&Wolpaw, J. R. (2008). Brain-computer interfaces in neurological rehabilitation. Lancet Neurol, Vol.7,No. 11, pp 1032-43

Delorme, A. \&Makeig, S. (2003). EEG changes accompanying learned regulation of $12-\mathrm{Hz}$ EEG activity. IEEE Trans Neural SystRehabilEng, Vol.11,No. 2, pp 133-7

Dietz, V., Quintern, J. \& Berger, W. (1984). Cerebral evoked potentials associated with the compensatory reactions following stance and gait perturbation. NeurosciLett, Vol.50,No. 1-3, pp 181-6

Dimitrijevic, M. R., Gerasimenko, Y. \& Pinter, M. M. (1998). Evidence for a spinal central pattern generator in humans.Ann NY AcadSci, Vol.860,, p 360-76

Dobkin, B. H. (2007). Brain-computer interface technology as a tool to augment plasticity and outcomes for neurological rehabilitation. J Physiol, Vol.579,No. 3, pp 637-42

Doyon, J. \&Benali, H. (2005). Reorganization and plasticity in the adult brain during learning of motor skills.CurrOpinNeurobiol, Vol.15,No. 2, pp 161-7

Drew, T., Prentice, S. \&Schepens, B. (2004). Cortical and brainstem control of locomotion. Prog Brain Res, Vol.143,, p 251-61

Duncan, P. W., Lai, S. M. \& Keighley, J. (2000). Defining post-stroke recovery: implications for design and interpretation of drug trials. Neuropharmacology, Vol.39,No. 5, pp $835-41$

Enzinger, C., Dawes, H., Johansen-Berg, H., Wade, D., Bogdanovic, M., Collett, J., Guy, C., Kischka, U., Ropele, S., Fazekas, F. \& Matthews, P. M. (2009). Brain activity changes associated with treadmill training after stroke. Stroke, Vol.40,No. 7, pp 2460-7 
Fox, P. T. \&Raichle, M. E. (1986). Focal physiological uncoupling of cerebral blood flow and oxidative metabolism during somatosensory stimulation in human subjects. ProcNatlAcadSci U S A, Vol.83,No. 4, pp 1140-4

Fuchs, T., Birbaumer, N., Lutzenberger, W., Gruzelier, J. H. \& Kaiser, J. (2003).Neurofeedback treatment for attention-deficit/hyperactivity disorder in children: a comparison with methylphenidate. ApplPsychophysiol Biofeedback, Vol.28,No. 1, pp 1-12

Fukuyama, H., Ouchi, Y., Matsuzaki, S., Nagahama, Y., Yamauchi, H., Ogawa, M., Kimura, J. \&Shibasaki, H. (1997). Brain functional activity during gait in normal subjects: a SPECT study. NeurosciLett, Vol.228,No. 3, pp 183-6

Furusho, J., Koyanagi, K., Imada, Y., Fujii, Y., Nakanishi, K., Domen, K., Miyakoshi, K., Ryu, U., Takenaka, S. \& Inoue, A. (2005). A 3-D Rehabilitation system for Upper Limbs. Developed in a 5-year NEDO Project and its Clinical Testing. Proceedings of IEEE 9th International Conference on Rehabilitation Robotics.

Grafton, S. T., Mazziotta, J. C., Presty, S., Friston, K. J., Frackowiak, R. S. \& Phelps, M. E. (1992). Functional anatomy of human procedural learning determined with regional cerebral blood flow and PET. J Neurosci, Vol.12,No. 7, pp 2542-8

Gratton, G., Maier, J. S., Fabiani, M., Mantulin, W. W. \&Gratton, E. (1994).Feasibility of intracranial near-infrared optical scanning.Psychophysiology, Vol.31,No. 2, pp 211-5

Grillner, S. \&Wallen, P. (2004). Innate versus learned movements--a false dichotomdddy? Prog Brain Res, Vol.143,pp 3-12

Hatakenaka, M., Miyai, I., Mihara, M., Sakoda, S. \& Kubota, K. (2007). Frontal regions involved in learning of motor skill--A functional NIRS study. Neuroimage, Vol.34,No. 1, pp 109-16

Jahn, K., Deutschlander, A., Stephan, T., Strupp, M., Wiesmann, M. \& Brandt, T. (2004). Brain activation patterns during imagined stance and locomotion in functional magnetic resonance imaging. Neuroimage, Vol.22,No. 4, pp 1722-31

Jenkins, W. M. \&Merzenich, M. M. (1987). Reorganization of neocortical representations after brain injury: a neurophysiological model of the bases of recovery from stroke. Prog Brain Res, Vol.71,, p 249-66

Jorgensen, H. S., Nakayama, H., Raaschou, H. O. \& Olsen, T. S. (1999). Stroke. Neurologic and functional recovery the Copenhagen Stroke Study.Phys Med RehabilClin N Am, Vol.10,No. 4, pp 887-906

Kohno, S., Miyai, I., Seiyama, A., Oda, I., Ishikawa, A., Tsuneishi, S., Amita, T. \& Shimizu, K. (2007).Removal of the skin blood flow artifact in functional near-infrared spectroscopic imaging data through independent component analysis.J Biomed Opt, Vol.12,No. 6, pp 062111

Kotchoubey, B., Strehl, U., Uhlmann, C., Holzapfel, S., Konig, M., Froscher, W., Blankenhorn, V. \&Birbaumer, N. (2001). Modification of slow cortical potentials in patients with refractory epilepsy: a controlled outcome study. Epilepsia, Vol.42,No. 3, pp 406-16

Luft, A. R., Forrester, L., Macko, R. F., McCombe-Waller, S., Whitall, J., Villagra, F. \& Hanley, D. F. (2005). Brain activation of lower extremity movement in chronically impaired stroke survivors. Neuroimage, Vol.26,No. 1, pp 184-94 
Maki, A., Yamashita, Y., Ito, Y., Watanabe, E., Mayanagi, Y. \& Koizumi, H. (1995).Spatial and temporal analysis of human motor activity using noninvasive NIR topography.Med Phys, Vol.22,No. 12, pp 1997-2005

Malouin, F., Richards, C. L., Jackson, P. L., Dumas, F. \& Doyon, J. (2003). Brain activations during motor imagery of locomotor-related tasks: a PET study. Hum Brain Mapp, Vol.19,No. 1, pp 47-62

Matsuyama, K., Mori, F., Nakajima, K., Drew, T., Aoki, M. \& Mori, S. (2004). Locomotor role of the corticoreticular-reticulospinal-spinal interneuronal system.Prog Brain Res, Vol.143,pp 239-49

Mihara, M., Miyai, I., Haraguchi, M., Kikuchi, T., Hatakenaka, M., Hattori, N. \&Furusho, J. (2009). Cortical network involved in the adaptation learning of reaching using 3dimensional robotic rehabilitation system: A functional near-infrared spectroscopic study. Neuroimage, Vol.47, Supplement 1, pp S170

Mihara, M., Miyai, I., Hatakenaka, M., Kubota, K. \&Sakoda, S. (2007). Sustained prefrontal activation during ataxic gait: a compensatory mechanism for ataxic stroke? Neuroimage, Vol.37,No. 4, pp 1338-45

Mihara, M., Miyai, I., Hatakenaka, M., Kubota, K. \&Sakoda, S. (2008). Role of the prefrontal cortex in human balance control.Neuroimage, Vol.43,No. 2, pp 329-36

Miyai, I., Suzuki, T., Kang, J., Kubota, K. \& Volpe, B. T. (1999). Middle cerebral artery stroke that includes the premotor cortex reduces mobility outcome. Stroke, Vol.30,No. 7, pp 1380-3

Miyai, I., Tanabe, H. C., Sase, I., Eda, H., Oda, I., Konishi, I., Tsunazawa, Y., Suzuki, T., Yanagida, T. \& Kubota, K. (2001). Cortical mapping of gait in humans: a nearinfrared spectroscopic topography study. Neuroimage, Vol.14,No. 5, pp 1186-92

Miyai, I., Yagura, H., Hatakenaka, M., Oda, I., Konishi, I. \& Kubota, K. (2003).Longitudinal optical imaging study for locomotor recovery after stroke.Stroke, Vol.34,No. 12, pp 2866-70

Miyai, I., Yagura, H., Oda, I., Konishi, I., Eda, H., Suzuki, T. \& Kubota, K. (2002). Premotor cortex is involved in restoration of gait in stroke. Ann Neurol, Vol.52,No. 2, pp 18894

Mori, S., Nakajima, K., Mori, F. \& Matsuyama, K. (2004). Integration of multiple motor segments for the elaboration of locomotion: role of the fastigial nucleus of the cerebellum. Prog Brain Res, Vol.143,, p 341-51

Morton, S. M. \& Bastian, A. J. (2004). Cerebellar control of balance and locomotion.Neuroscientist, Vol.10,No. 3, pp 247-59

Nielsen, J. B. (2003). How we walk: central control of muscle activity during human walking. Neuroscientist, Vol.9,No. 3, pp 195-204

Nudo, R. J., Wise, B. M., SiFuentes, F. \& Milliken, G. W. (1996).Neural substrates for the effects of rehabilitative training on motor recovery after ischemic infarct.Science, Vol.272,No. 5269, pp 1791-4

O'Loughlin, J. L., Robitaille, Y., Boivin, J. F. \&Suissa, S. (1993). Incidence of and risk factors for falls and injurious falls among the community-dwelling elderly.Am J Epidemiol, Vol.137,No. 3, pp 342-54

Okamoto, M., Dan, H., Sakamoto, K., Takeo, K., Shimizu, K., Kohno, S., Oda, I., Isobe, S., Suzuki, T., Kohyama, K. \& Dan, I. (2004). Three-dimensional probabilistic 
anatomical cranio-cerebral correlation via the international 10-20 system oriented for transcranial functional brain mapping. Neuroimage, Vol.21,No. 1, pp 99-111

Quant, S., Maki, B. E. \&Mcllroy, W. E. (2005).The association between later cortical potentials and later phases of postural reactions evoked by perturbations to upright stance.NeurosciLett, Vol.381,No. 3, pp 269-74

Sitaram, R., Zhang, H., Guan, C., Thulasidas, M., Hoshi, Y., Ishikawa, A., Shimizu, K. \&Birbaumer, N. (2007). Temporal classification of multichannel near-infrared spectroscopy signals of motor imagery for developing a brain-computer interface. Neuroimage, Vol.34,No. 4, pp 1416-27

Slobounov, S., Hallett, M., Stanhope, S. \&Shibasaki, H. (2005). Role of cerebral cortex in human postural control: an EEG study. ClinNeurophysiol, Vol.116,No. 2, pp 315-23

Suzuki, M., Miyai, I., Ono, T., Oda, I., Konishi, I., Kochiyama, T. \& Kubota, K. (2004). Prefrontal and premotor cortices are involved in adapting walking and running speed on the treadmill: an optical imaging study. Neuroimage, Vol.23,No. 3, pp 1020-6

Takahashi, T., Takikawa, Y., Kawagoe, R., Shibuya, S., Iwano, T. \& Kitazawa, S. (2011). Influence of skin blood flow on near-infrared spectroscopy signals measured on the forehead during a verbal fluency task. Neuroimage, Vol.57,No. 3, pp 991-1002

Takakusaki, K., Oohinata-Sugimoto, J., Saitoh, K. \&Habaguchi, T. (2004). Role of basal ganglia-brainstem systems in the control of postural muscle tone and locomotion.Prog Brain Res, Vol.143,pp 231-7

Ward, N. \&Frackowiak, R. (2004). The cerebral basis of functional recovery., In. Human brain function. F. K. Frackowiak RSJ, Frith CD, Dolan RJ, Price CJ, Zeki S, Ashburner J, Penny W. pp. 105-123, Academic press.San Diego

Ward, N. S., Brown, M. M., Thompson, A. J. \&Frackowiak, R. S. (2003). Neural correlates of motor recovery after stroke: a longitudinal fMRI study. Brain, Vol.126,Pt 11, pp 2476-96

Woollacott, M. \& Shumway-Cook, A. (2002). Attention and the control of posture and gait: a review of an emarging area of research. Gait Posture, Vol.16,pp1-14

Yamada, T., Umeyama, S. \& Matsuda, K. (2009).Multidistanceprobe arrangement to eliminate artifacts in functional near-infrared spectroscopy. Journal of Biomedical Optics, Vol.14,No. 6, pp 064034 


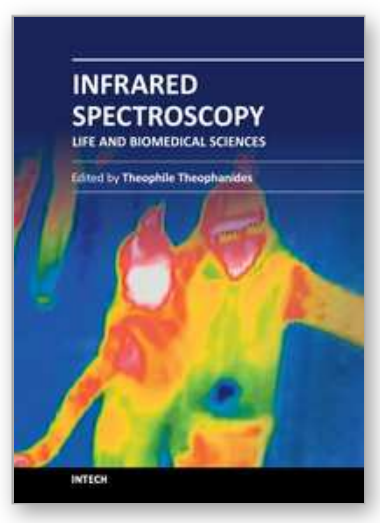

\author{
Infrared Spectroscopy - Life and Biomedical Sciences \\ Edited by Prof. Theophanides Theophile
}

ISBN 978-953-51-0538-1

Hard cover, 368 pages

Publisher InTech

Published online 25, April, 2012

Published in print edition April, 2012

This informative and state-of-the art book on Infrared Spectroscopy in Life sciences designed for researchers, academics as well as for those working in industry, agriculture and in pharmaceutical companies features 20 chapters of applications of MIRS and NIRS in brain activity and clinical research. It shows excellent FT-IR spectra of breast tissues, atheromatic plaques, human bones and projects assessment of haemodynamic activation in the cerebral cortex, brain oxygenation studies and many interesting insights from a medical perspective.

\title{
How to reference
}

In order to correctly reference this scholarly work, feel free to copy and paste the following:

Masahito Mihara and Ichiro Miyai (2012). Applications of Near Infrared Spectroscopy in Neurorehabilitation, Infrared Spectroscopy - Life and Biomedical Sciences, Prof. Theophanides Theophile (Ed.), ISBN: 978-953-510538-1, InTech, Available from: http://www.intechopen.com/books/infrared-spectroscopy-life-and-biomedicalsciences/applications-of-near-infrared-spectroscopy-in-neurorehabilitation

\section{INTECH}

open science | open minds

\section{InTech Europe}

University Campus STeP Ri

Slavka Krautzeka 83/A

51000 Rijeka, Croatia

Phone: +385 (51) 770447

Fax: +385 (51) 686166

www.intechopen.com

\section{InTech China}

Unit 405, Office Block, Hotel Equatorial Shanghai

No.65, Yan An Road (West), Shanghai, 200040, China

中国上海市延安西路65号上海国际贵都大饭店办公楼 405 单元

Phone: +86-21-62489820

Fax: $+86-21-62489821$ 
(C) 2012 The Author(s). Licensee IntechOpen. This is an open access article distributed under the terms of the Creative Commons Attribution 3.0 License, which permits unrestricted use, distribution, and reproduction in any medium, provided the original work is properly cited. 